\title{
Betreff: Umsetzung gemeinsame Pflegeausbildung
}

Auszug aus dem Brief des BLGS an die Mitglieder des Ausschusses für Gesundheit und für Familie, Senioren, Frauen und Jugend vom 08.09.2016.

Sehr geehrte Damen und Herren,

mit Bestürzung nehmen wir zur Kenntnis, dass sich die politische Entscheidung zur Neugestaltung der Ausbildung in den Pflegeberufen verzögert. Gleichzeitig erreichen uns in den einzelnen Bundesländern Signale, dass über Ausbildungsmodelle nachgedacht wird, die erneut in einer Spezialisierung nach „altem Muster" münden sollen.

Der BLGS vereint aus allen Bundesländern Pflegeschulen aller drei Fachrichtungen. Wir beschäftigen uns seit Jahren mit der Umsetzung einer generalistischen Pflegeausbildung. Wir bitten Sie um Ihre Aufmerksamkeit für dieses Schreiben. Wir bitten Sie um Anerkennung unserer Fachexpertise.

Eines der Kernargumente gegen die Generalistik bezieht sich auf die Unterschiede in den einzelnen Pflegesettings, denn es mache ja einen „Unterschied, ob ein Frühchen mit weniger als $500 \mathrm{~g}$ oder ein hochbetagter Mensch mit vaskulärem Syndrom vom Typ Alzheimer zu pflegen ist" (aus dem Plenarprotokoll 18/121 des Schleswig-Holsteinischen Landtags: 10081). Nach der Logik der Generalistikgegner dürfte keine Ärztin ein Frühgeborenes betreuen und kein Ergotherapeut einen hochbetagten Menschen unterstützen, denn alle Angehörigen der Gesundheits- und Heilberufe - außer der Pflege - sind Generalisten!

Die angeblich zu erwartenden Qualitätsmängel beruhen auf einem fundamentalen Missverständnis: Es geht bei der Schaffung eines einheitlichen Pflegeberufs nicht darum, die jetzigen Inhalte der drei Ausbildungsgänge einfach irgendwie zu vereinheitlichen. Vielmehr soll ein neues Pflegeberufsbild geschaffen werden, das die Versorgungsschwerpunkte der Menschen in allen Lebensaltersstufen abbildet. Ziel ist die Vermittlung von Kernkompetenzen in der Pflege, die die Auszubildenden und Studierenden auf alle pflegerischen Settings vorbereitet. Die notwendigen Qualifizierungen für hochspezialisierte berufliche Handlungsfelder finden im Rahmen der Berufstätigkeit und durch Fort- und Weiterbildung statt. Damit soll nun endlich auch die Pflege in die deutsche und internationale berufliche Bildungsnormalität überführt werden. Eine Beibehaltung der jetzigen drei Ausbildungsabschlüsse ist aus vielen Gründen nicht sinnvoll:

- Eine Pflegeausbildung, die komplexe Pflegephänomene bloß aus der Perspektive des Lebensalters vertieft, entspricht längst nicht mehr den heutigen pflegewissenschaftlichen und pflegedidaktischen Erkenntnissen. Settingstrukturen, Er-gebnisse der Versorgungsforschung und gesundheitswissenschaftliche Erkenntnisse würden außer Acht gelassen. Eine solche Ausrichtung würde einen althergebrachten Unterrichtsstil provozieren, der uns in Bildungsstrukturen der 70/80er Jahre zurückwirft.

- Die dreigeteilte Struktur der Pflegeausbildung ist der historischen Entwicklung der deutschen Sozialversicherungssysteme bzw. der Anbindung an politische Zuständigkeiten entsprungen. Sie entbehrt zunehmend einer inhaltlichen Sinnhaftigkeit und gefährdet immer stärker die angemessene pflegerische Versor- gung unserer Bevölkerung - vor allem auch der alten Menschen. Um mit dem europäischen Standard Schritt halten zu können, müssen Spezialisierungen im weiteren Verlauf der Berufstätigkeit im Rahmen beruflicher Weiterbildung oder eines Masterstudiums ausgebildet werden.

- Die Sorge um die "Verflachung der Ausbildung” bzw. „untaugliche Absolvent/innen für den Arbeitsmarkt" ist sachlich unbegründet. Wir bitten nachdrücklich, die wissenschaftliาchen Erkenntnisse aus den Modellversuchen endlich ernsthaft zur Kenntnis zu nehmen. Im Rahmen von "Transfernetzwerk innovative Pflegeausbildung" und "Pflegeausbildung in Bewegung" wurde deutlich gezeigt, dass die Absolvent/innen vom Arbeitsmarkt gut abgenommen werden konnten.

- Pflegetheoretische Grundlagen, die das fachliche Fundament der Ausbildung bilden, orientieren sich zu Recht nicht vorrangig am Konzept des Lebensalters, sondern stehen im Kontext Mensch - Umwelt - Gesundheit/Krankheit. Deutschland würde mit einer integrierten, weiterhin dreigeteilten Pflegeausbildung gegen den Stand wissenschaftlicher Erkenntnisse sowohl der Pflegewissenschaft als auch der Pflegedidaktik handeln.

- Die dreigliedrige Berufsstruktur hat den Pflegenden gravierende Chancenungleichheiten auf dem deutschen und internationalen Arbeitsmarkt beschert. Dazu gehören insbesondere ungerechte Vergütungsstrukturen und Einschränkungen in der Berufsausübung: So dürfen in manchen Bundesländern Altenpflegekräfte keine Sozialstationen leiten und nicht an Intensivund Anästhesieweiterbildungen teilnehmen. Mit der Kinderkrankenpflege und der Altenpflege sind zwei von drei deutschen Pflegeberufen europäisch nicht anerkannt. Ein neues Pflegeberufsgesetz, welches weiter die traditionelle Dreigliederung bzw. integrative Pflegeausbildung festschreibt, würde solche und andere Ungerechtigkeiten manifestieren, einen großen Teil der Pflegenden von bestimmten Karriereoptionen ausschließen und insgesamt die europäische Anerkennung gefährden.

Viele Pflegeschulen vereinen längst mehrere Pflegeausbildungsgänge unter einem Dach oder kooperieren untereinander. Schulen sind in den letzten Jahren in neue Rechtsformen überführt worden und warten nur darauf, den letzten Schritt tun zu können. Wir bitten Sie um Mut zur Umsetzung der gemeinsamen Grundausbildung - noch in diesem Jahr.

Wir verstehen unser Geschäft. Wir bilden täglich aus und tragen die Verantwortung für die Ausbildung von heute und morgen. Wir wissen um die Sorgen, die mit der Einführung der neuen Strukturen einhergehen. Auch für jede unserer Schulen wird sich vieles verändern. Wir wissen aber auch, was die pflegerische Fachpraxis benötigt, um in Zukunft die Menschen pflegerisch versorgen zu können. Wir haben den Mut, die Veränderungen umzusetzen. Geben Sie uns das nötige Gesetz in die Hand.

Wir freuen uns auf die Zusammenarbeit mit Ihnen, um den Weg für eine zukunftsorientierte Ausbildung gemeinsam gestalten zu können. 